\section{(1) \\ CrossMark}

\title{
Pneumocystosis revealing immunodeficiency secondary to TERC mutation
}

\author{
To the Editor:
}

Telomerase-related gene (TRG) mutations are evidenced in about $25 \%$ of patients with familial pulmonary fibrosis, and less frequently in sporadic interstitial lung disease (ILD) [1]. Even though TRG mutations can be associated with haematological, hepatic and cutaneous manifestations, most adult ILD patients with TRG mutations only present asymptomatic extrapulmonary involvement such as thrombocytopenia or premature hair greying [2]. However, some specific extrapulmonary manifestations such as immunodeficiency or portal hypertension can be severe and need early detection. We report herein the case of a patient with primary immunodeficiency revealed by pneumocystosis, which could have been misdiagnosed as an idiopathic acute exacerbation of ILD.

A 47-year-old female non-smoker was admitted to our department for acute respiratory insufficiency. Her medical history revealed chronic asymptomatic neutropenia (between 1000 and $1500 \mathrm{~mm}^{-3}$ ), thrombocytopenia (between 100000 and $150000 \mathrm{~mm}^{-3}$ ) and a premature occurrence of white hair at the age of 19 years. Apart from her 18-year-old son who with presented thrombocytopenia $\left(<50000 \mathrm{~mm}^{-3}\right)$, neutropenia and splenomegaly without diagnosis, the other members of her family were perfectly healthy.

6 months before admission, she had presented shortness of breath and a computed tomography (CT) scan showed upper-lobe subpleural consolidations with lung volume loss evocative of pleuro-parenchymal fibroelastosis (PPFE) (figure 1a). The pulmonary function tests showed mild defects with forced vital capacity (FVC) at $84 \%$ and diffusing lung capacity for carbon monoxide (DLCO) at $68 \%$ of the predicted values. At that time the patient refused complementary examinations and treatment.

6 months later, she presented with acute respiratory deterioration for 2 weeks without fever or increased sputum production and required hospitalisation. Clinical examination revealed bilateral crackles, numerous warts located on her hands and feet, associated with skin hyperpigmentation, dysplastic nails and oral leukoplakia, the characteristic triad of dyskeratosis congenita. The clinical diagnosis of dyskeratosis congenita was ascertained on the presence of the triad, the premature hair greying and pulmonary disease. FVC was $62 \%$ and DLCO $40 \%$ of the predicted values. A CT scan showed patchy ground-glass opacities and a few new consolidations superimposed with previously known ILD (figure 1b).

A blood test showed normal haemoglobin and platelet counts. The lymphocyte count was $790 \mathrm{~mL}^{-1}$, CD3 at $480 \mathrm{~mm}^{-3}$ (49.3\%), CD4 at $285 \mathrm{~mm}^{-3}$ (29.3\%), CD8 at $200 \mathrm{~mm}^{-3}(20.4 \%)$ and B cells at $394 \mathrm{~mm}^{-3}$ (40.2\%). HIV testing was negative.

An acute exacerbation of PPFE was initially suspected and a bronchoalveolar lavage (BAL) was performed to exclude an infection. Cytological examination of the BAL fluid revealed numerous cysts of Pneumocytis jirovecii at direct examination and PCR was positive at 2058857 copies $\cdot \mathrm{mm}^{-3}$ without any other infection.

Because of the personal and familial history of haematological abnormalities, a genetic analysis of TERT and TERC was performed. Genetic analysis showed in both patient and son a heterozygous variant of TERC, r.448A>U, (NR_001566 : hg19 : chr3 : 169482401_T_A) which has never been reported before (http://telomerase.asu.edu/diseases.html). This variant is absent from the gnomAD browser, which includes exome sequencing data from $>80000$ individuals (http:/gnomad.broadinstitute.org/gene/ ENSG00000270141). It is located in the ACA box, a highly conserved region of TERC and the GERP score (an in silico score reflecting nucleotide-level sequence conservation) is high (5.32), suggesting a causal 13993003.01443-2017]. 

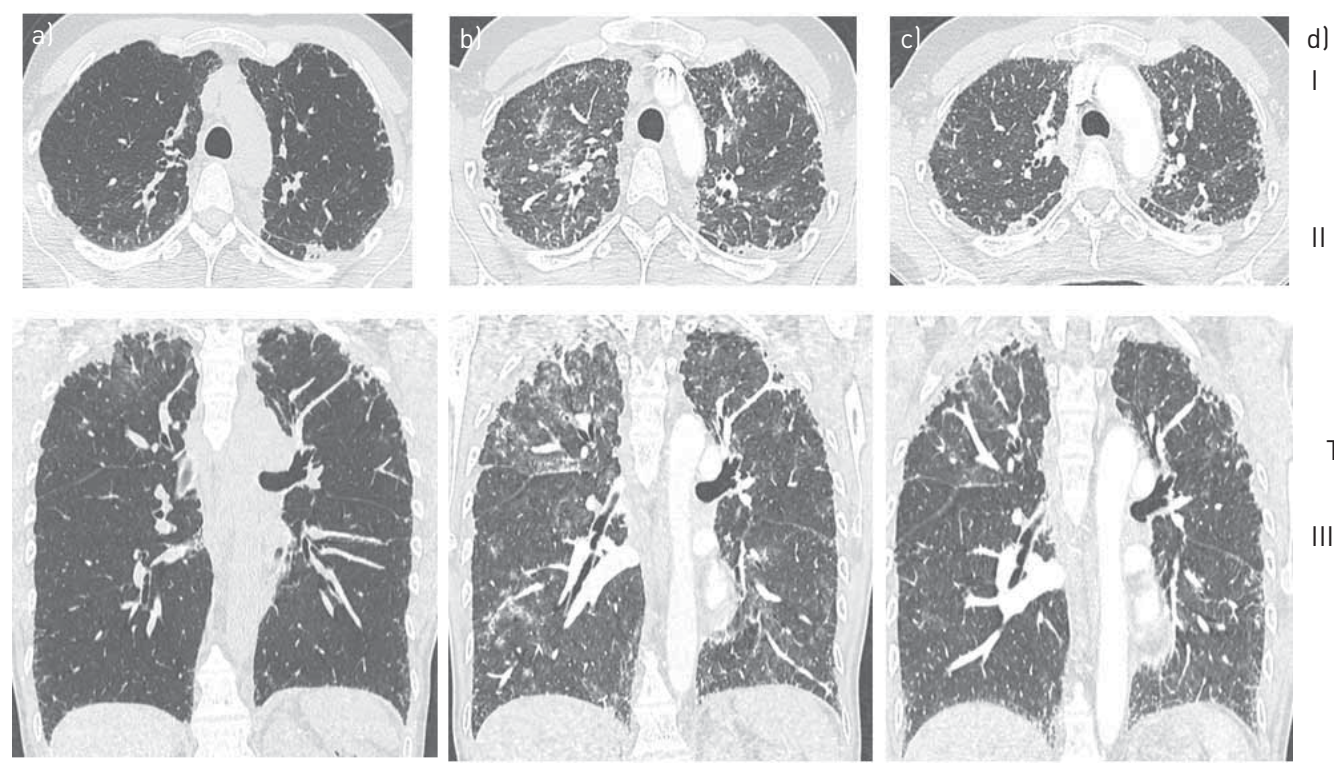

d)
II

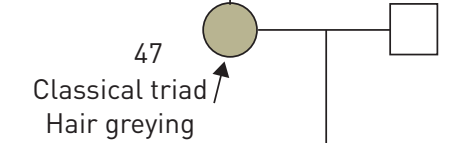

Neutropenia

Thrombocytopenia

PPFE

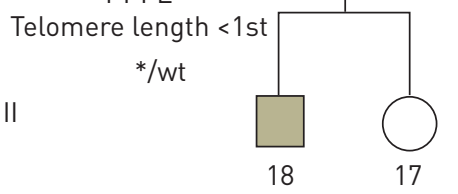

Thrombocytopenia wt/wt

Neutropenia

Splenomegaly

$* /$ wt

FIGURE 1 Chest computed tomography scan performed a) 6 months before the pneumocystosis with a pattern suggestive of pleuropulmonary fibroelastosis, b) at the time of pneumocystosis with superimposed ground-glass opacities and c) 2 months after initiation of cotrimoxazole therapy, showing impressive decrease of ground-glass opacities. d) Pedigree of the whole family. TERC genetic analysis is indicated as wt/wt in the absence of the mutation and */wt for heterozygous carrier of the mutation. Arabic numbers indicate age at proband diagnosis. Telomere length less than first percentile was obtained by flow-fluorescent in situ hybridisation. Individuals in grey display clinical features of dyskeratosis congenita described below.

variant. Moreover, this variant was identified in two other unrelated families with aplastic anaemia (unpublished data). This variant was absent in her healthy daughter and in her healthy parents. Maternity and paternity was confirmed using the PowerPlex 16 System from Promega. Finally, although the functional effect of the variant was not specifically evaluated, the blood leukocyte telomere length of the patient analysed by flow-fluorescent in situ hybridisation was below the first percentile, again favouring a pathogenic variant. Altogether the variant meets the ACMG criteria of pathogenicity: de novo mutation of TERC [3].

The patient received high dose steroids for 3 days and 3 weeks of curative cotrimoxazole, which had to be suspended for a week because of transient cytopenia. She was then able to receive a lower dose of cotrimoxazole as a prophylactic therapy, without adverse event. A treatment with nintedanib $150 \mathrm{mg}$ twice daily and danazol $400 \mathrm{mg}$ twice daily was also introduced, leading to progressive improvement.

Indeed, the platelet count increased to 174000 , haemoglobin level to $12.9 \mathrm{~g} \cdot \mathrm{dL}^{-1}$ and neutrophils to $2700 \mathrm{~mm}^{-3}$. Of note, in the National Institutes of Health prospective trial, the mean increase in haemoglobin was $3.3 \mathrm{~g} \cdot \mathrm{dL}^{-1}$ and $300 \mathrm{~mm}^{-3}$ for neutrophils and 14250 for platelets [3].

1 year after the diagnosis of pneumocystosis, the patient was able to work; the FVC was at $87 \%$ and DLCO at $54 \%$ of the predicted values. The last available CT scan showed a significant decrease in ground-glass opacities compared with the CT scan performed during pneumocystosis (figure 1c).

This case report highlights the heterogeneity of manifestations related to telomere syndrome. Indeed, with a focus on pulmonary manifestations, patients may present with pulmonary fibrosis and hepatopulmonary syndrome, and have an increased risk of opportunistic infections $[2,4,5]$.

ILD is the most frequent pulmonary manifestation and up to $60 \%$ of the patients with the TERT mutation will present pulmonary fibrosis at the age of 60 years [6]. Usually, interstitial pneumonia is the most frequent pattern undistinguishable from IPF, but the frequency of the PPFE pattern also appears to be high $[4,7]$. However, and as far as we know, TERC mutation, dyskeratosis congenita and PPFE occurring in the same patient has never been reported before. In a retrospective study, neither the CT pattern nor the gene involved (TERT, TERC, PARN or RTEL1) was reported to impact the decline of FVC or the survival [4]. Indeed, because of the young age of the patient, and irreversible PPFE with a high risk of chronic respiratory insufficiency, we prescribed off-label oral nintedanib, although nintedanib has never been evaluated in TRG mutation carriers or PPFE.

TRG mutations are also associated with hepatic and vascular diseases which may be responsible for the development of hepatopulmonary syndrome with hypoxaemia or intrapulmonary shunt, as reported in almost 20 patients with TERT, TINF2, PARN RTEL1 or DKC1 mutations [5, 8]. 
TERC and DKC1 mutations were initially reported in dyskeratosis congenita, a syndrome defined by reticular skin pigmentation, nail dystrophy and oral mucosal leukoplakia. Bone marrow failure usually appears during the second decade of life, as well as lung fibrosis. Dyskeratosis congenita is nowadays clearly identified as a cause of immunodeficiency including lymphopenia, low B-cell numbers, hypogammaglobulinaemia and decreased T-cell function $[9,10]$. Patients with dyskeratosis congenita are particularly at risk for pneumocystis. However, following lung transplantation, patients with lung fibrosis and the TRG mutation did not appear to present a higher risk of opportunistic infection [11-13].

Unlike most patients previously reported with the TRG mutation and ILD, the patient herein fulfilled the dyskeratosis congenita criteria. The cutaneous triad and the presence of numerous warts led us to suspect an opportunistic infection, although an idiopathic acute exacerbation was our first diagnosis in the absence of immunosuppressive therapy. In this case, a specific reduced total and CD4 lymphocyte counts may explain opportunistic infection, which are unexpected features in sporadic dysmyelopoiesis.

This observation supports that TRG mutation may be specifically associated with an increased risk of Pneumocystis infection. In any case, before any conclusion of idiopathic acute exacerbation, a diagnostic test should be performed to exclude opportunistic infection in confirmed or suspected TRG mutation carriers. Finally the patient improved with danazol, which has been shown to ameliorate blood counts in the clinic and should be further evaluated in that context.

Raphael Borie ${ }^{1,2}$, Caroline Kannengiesser $\oplus^{3}$, Flore Sicre de Fontbrune ${ }^{4}$, David Boutboul ${ }^{5}$, Laure Tabeze ${ }^{1}$, Florence Brunet-Possenti ${ }^{6}$, Elodie Lainey ${ }^{7}$, Marie Pierre Debray ${ }^{8}$, Aurélie Cazes ${ }^{2,9}$ and Bruno Crestani ${ }^{1,2}$

${ }^{1}$ APHP, Hôpital Bichat, Service de Pneumologie A, Centre de référence des maladies pulmonaires rares, Paris, France. ${ }^{2}$ INSERM, Unité 1152, Université Paris Diderot, Paris, France. ${ }^{3}$ Laboratoire de Génétique, APHP, Hopital Bichat, Université Paris Diderot, Paris, France. ${ }^{4}$ Service d'Hématologie greffe, Centre de référence maladie rare aplasie médullaire, APHP, Hôpital St Louis, Paris, France. ${ }^{5}$ INSERM UMR 1163, Laboratory of Genome Dynamics in the Immune System, Paris Descartes - Sorbonne Paris Cité University, Imagine Institute, Paris, France. ${ }^{6}$ APHP, Hôpital Bichat, Service de Dermatologie, Paris, France. ${ }^{7}$ APHP, Hopital Robert Debré, Laboratoire d’hématologie, Paris, France. ${ }^{8}$ APHP, Hôpital Bichat, Laboratoire de radiologie, Paris, France. ${ }^{9}$ APHP, Hôpital Bichat, Laboratoire d'anatomopathologie, Paris, France.

Correspondence: Raphael Borie, Service de pneumologie A, Hôpital Bichat, 46 rue Henri Huchard, 75877 Paris CEDEX 18, France. E-mail: raphael.borie@aphp.fr

Received: July 172017 | Accepted after revision: Sept 012017

Support statement: Funded by F. Hoffmann-La Roche and Legs Poix. Funding information for this article has been deposited with the Crossref Funder Registry.

Conflict of interest: Disclosures can be found alongside this article at erj.ersjournals.com

This article has been revised according to the correction published in the February 2018 issue of the European Respiratory Journal.

\section{References}

1 Borie R, Kannengiesser C, Sicre de Fontbrune F, et al. Management of suspected monogenic lung fibrosis in a specialised centre. Eur Respir Rev 2017; 26: 160122.

2 Borie R, Tabèze L, Thabut G, et al. Prevalence and characteristics of TERT and TERC mutations in suspected genetic pulmonary fibrosis. Eur Respir J 2016; 48: 1721-1731.

3 Townsley DM, Dumitriu B, Liu D, et al. Danazol treatment for telomere diseases. N Engl J Med 2016; 374: 1922-1931.

4 Newton CA, Batra K, Torrealba J, et al. Telomere-related lung fibrosis is diagnostically heterogeneous but uniformly progressive. Eur Respir J 2016; 48: 1710-1720.

5 Gorgy AI, Jonassaint NL, Stanley SE, et al. Hepatopulmonary syndrome is a frequent cause of dyspnea in the short telomere disorders. Chest 2015; 148: 1019-1026.

6 Diaz de Leon A, Cronkhite JT, Katzenstein AL, et al. Telomere lengths, pulmonary fibrosis and telomerase (TERT) mutations. PLoS One 2010; 5: e10680.

7 Nunes H, Jeny F, Bouvry D, et al. Pleuroparenchymal fibroelastosis associated with telomerase reverse transcriptase mutations. Eur Respir J 2017; 49: 1602022.

8 Khincha PP, Bertuch AA, Agarwal S, et al. Pulmonary arteriovenous malformations: an uncharacterised phenotype of dyskeratosis congenita and related telomere biology disorders. Eur Respir J 2017; 49: 1601640.

9 Jyonouchi S, Forbes L, Ruchelli E, et al. Dyskeratosis congenita: a combined immunodeficiency with broad clinical spectrum-a single-center pediatric experience. Pediatr Allergy Immunol 2011; 22: 313-319.

10 Bousfiha A, Jeddane L, Al-Herz W, et al. The 2015 IUIS phenotypic classification for primary immunodeficiencies. J Clin Immunol 2015; 35: 727-738.

11 Borie R, Kannengiesser C, Hirschi S, et al. Severe hematologic complications after lung transplantation in patients with telomerase complex mutations. J Heart Lung Transplant 2015; 34: 538-546.

12 Silhan LL, Shah PD, Chambers DC, et al. Lung transplantation in telomerase mutation carriers with pulmonary fibrosis. Eur Respir J 2014; 44: 178-187.

13 Tokman S, Singer JP, Devine MS, et al. Clinical outcomes of lung transplant recipients with telomerase mutations. J Heart Lung Transplant 2015; 34: 1318-1324. 